\title{
Solidification of undercooled peritectic Fe-Ge alloy
}

\author{
Gandham Phanikumar ${ }^{\mathrm{a}, *, 1}$, Krishanu Biswas ${ }^{\mathrm{b}}$, Oliver Funke ${ }^{\mathrm{a}}$, Dirk Holland-Moritz ${ }^{\mathrm{a}}$, \\ Dieter M. Herlach ${ }^{a}$, Kamanio Chattopadhyay ${ }^{b}$ \\ ${ }^{a}$ Institute of Space Simulation, German Aerospace Center (DLR), Linder Hoehe, 51147 Cologne, Germany \\ ${ }^{\mathrm{b}}$ Department of Metallurgy, Indian Institute of Science, Bangalore 560 012, India
}

\begin{abstract}
Bulk samples of $\mathrm{Fe}-25$ at.\% Ge peritectic alloy are undercooled up to $260 \mathrm{~K}$ using electromagnetic levitation technique. The growth rate of the primary phase is measured using a capacitance proximity sensor technique. Solidification microstructure is studied as a function of undercooling. The microstructure of samples at low undercoolings consists of a residual primary phase $\alpha_{2}$, peritectic phase $\varepsilon$ and inter-dendritic $\varepsilon-\beta$ eutectic. Microstructure at higher undercoolings is nearly phase-pure $\varepsilon$. Time resolved diffraction analysis of the levitated droplets using synchrotron radiation indicates the nucleation of primary $\alpha_{2}$ in all cases. The growth rate is analysed using current theories to explain the experimental observations. Interfacial undercooling is found to play an important role in the growth kinetics. Our results also suggest suppression of peritectic reaction.
\end{abstract}

Keywords: Fe-Ge; Peritectic; Dendrite growth rate; Undercooling; Microstructure

\section{Introduction}

Solidification of undercooled melts enables study of crystal growth phenomena under non-equilibrium conditions. Deviation of chemical equilibrium at the solid-liquid interface and kinetic undercooling play significant roles in metastable phase formation [1].

The binary system Fe-Ge (Fig. 1) [2] comprises several critical points and order-disorder phase transitions. However, studies on this system are limited [3-5]. The intermetallic phase $\mathrm{Fe}_{3} \mathrm{Ge}$ is of interest for its mechanical properties [6,7]. The alloy composition chosen for

\footnotetext{
* Corresponding author. Tel.: +49 2203 6012428; fax: +49 2203 6012255.

E-mail addresses: g_phanikumar@yahoo.com, gphani@iitm.ac.in (G. Phanikumar).

${ }^{1}$ Present address: Department of Metallurgical and Materials Engineering, Indian Institute of Technology Madras, Chennai 600036, India.
}

the study is indicated by an arrow in Fig. 1 [2]. According to the equilibrium phase diagram, the alloy composition chosen undergoes a peritectic reaction, namely, $\mathrm{L}+\alpha_{2} \rightarrow \varepsilon$. The alloy solidifies with nucleation of ordered phase $\alpha_{2}(\mathrm{~B} 2)$ that reacts with the remaining liquid to form the peritectic ordered phase $\varepsilon\left(\mathrm{DO}_{19}\right)$. Peritectic reaction is controlled by diffusion across the product phase and usually does not proceed to completion and leads to a phase mixture in the final microstructure [8].

The $\varepsilon$-phase is dimorphic and the transformation from $\varepsilon \rightarrow \varepsilon^{\prime}\left(\mathrm{L1}_{2}\right)$ is sluggish [9]. Attention is also drawn to the eutectic reaction $\mathrm{L} \rightarrow \varepsilon+\beta$ close to the composition chosen for this study. Rapid solidification using the undercooling technique provides a possibility for suppressing the peritectic reaction and solute partitioning to obtain phase pure $\varepsilon$ [5]. Further, the sluggish nature of the transformation $\varepsilon \rightarrow \varepsilon^{\prime}$ can enable the metastable $\varepsilon$ to be retained at room temperature. The present study is aimed at determining quantitatively the kinetics of primary solidification and the evolution of microstructure 


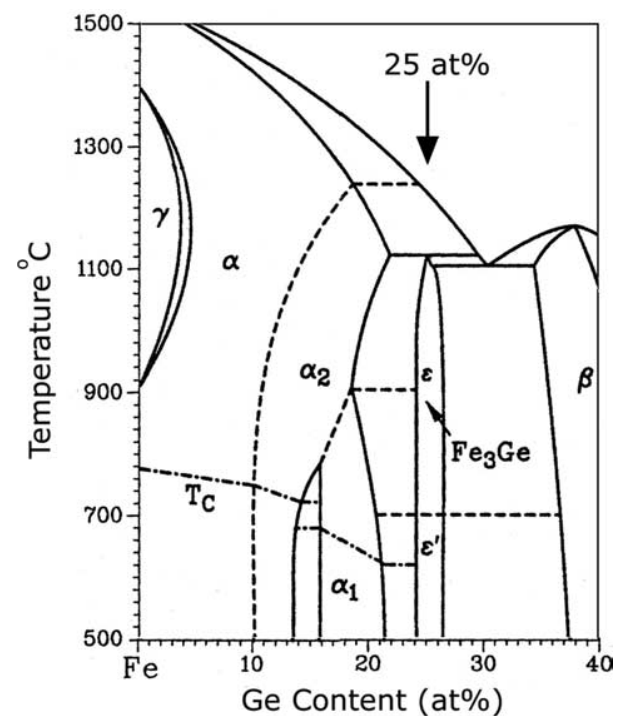

Fig. 1. Binary alloy phase diagram of $\mathrm{Fe}-\mathrm{Ge}$ system [4].

as a function of undercooling to understand the solidification behaviour of alloys with large solute concentration and ordering tendencies in solid state.

\section{Experiments}

The experiments were carried out using an electromagnetic levitation facility [10]. Samples of nominal composition $\mathrm{Fe}_{75} \mathrm{Ge}_{25}$ were prepared from elemental components of purity better than $99.99 \%$ using an arc furnace. Each sample of about $1.1 \mathrm{~g}$ weight and 6-8 $\mathrm{mm}$ in diameter was inserted into the levitation coil in the chamber and held using an alumina sample holder. The chamber was evacuated to a pressure of $10^{-6} \mathrm{mbar}$ and refilled to $10^{3}$ mbar pressure using a protective gas $\left(\mathrm{He}+5 \mathrm{vol} . \% \mathrm{H}_{2}\right)$ of $99.9999 \%$ purity. After the sample was levitated, the sample holder was withdrawn to keep the sample in a containerless environment. The sample was heated by induction to temperatures above the liquidus, held for few minutes to homogenize it and then undercooled by blowing the protective gas on to the sample. The temperature was measured using a two-colour pyrometer with an accuracy of $\pm 5 \mathrm{~K}$ and a measurement frequency of $100 \mathrm{~Hz}$.

A typical cooling and heating cycle is shown in Fig. 2. The cooling rate achieved by blowing gas was about $10 \mathrm{~K} / \mathrm{s}$. The solidification of undercooled sample was stimulated by touching the sample at the bottom using a needle of $99.99 \% \mathrm{Fe}$ at a predetermined temperature $\left(T_{\mathrm{N}}\right)$. The bulk undercooling $(\Delta T)$ achieved the difference between the liquidus $\left(T_{\mathrm{L}}\right)$ and the nucleation temperature $\left(T_{\mathrm{N}}\right)$. The sudden rise in temperature signal (indicated in the figure by an arrow and labelled " 1 st") detected by the pyrometer indicates the release of latent heat due to solidification and is termed "recalescence". A second

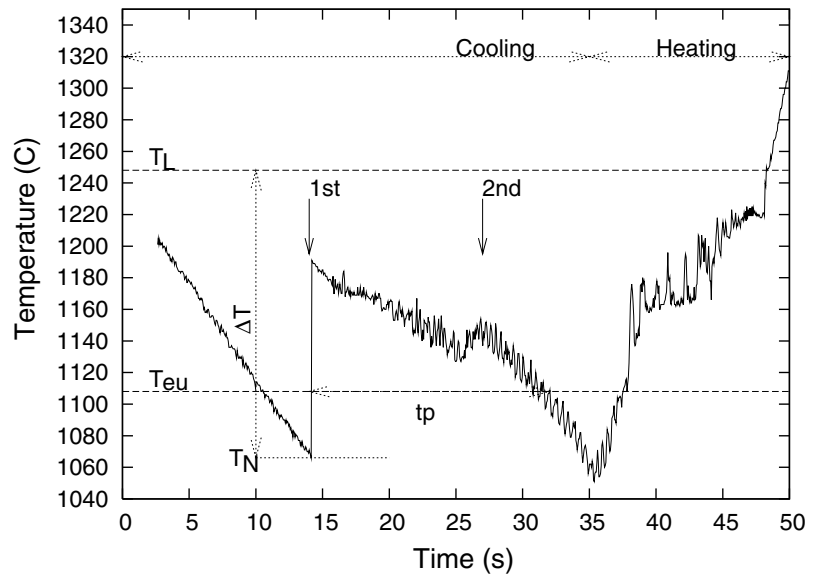

Fig. 2. Typical time-temperature cycle for the system.

such signal (labelled "2nd") is due to the formation of $\varepsilon$ phase. The second signal is usually weak and is often difficult to distinguish from the undulations in the pyrometer signal. The temperature of the last liquid to solidify, i.e., solidus/eutectic, is also indicated $\left(T_{\text {eu }}\right)$. The time spent by the sample between $T_{\mathrm{N}}$ and $T_{\text {eu }}$ is referred to as the plateau duration $\left(t_{\mathrm{p}}\right)$.

The trigger needle is also part of a capacitance proximity sensor (CPS) [11] that produces a distinct voltage signal coinciding with the triggering event. The end of solidification was detected by a photodiode that observes the sample from the top. The time resolved voltage signals from the CPS and photodiode, both of accuracy better than $10 \mu \mathrm{s}$, were measured by a 400 $\mathrm{MHz}$ digital oscilloscope. The time difference between the two signals gives the total solidification time. Complete details of the growth rate measurement setup are provided elsewhere [12]. Samples were solidified at different undercoolings as described above and cooled off with the protective gas to room temperature and taken out for characterization.

As-solidified samples were sectioned for further characterization using X-ray diffraction (XRD), optical microscopy (OM, Olympus), scanning electron microscopy (SEM, LEO1530VP) equipped with a field emission gun, energy dispersive X-rays (EDS, Oxford) and transmission electron microscopy (TEM, JEOL 2000 FXII). XRD was performed using Fe K $\alpha$ radiation with a wavelength of $\lambda=0.1937 \mathrm{~nm}$. SEM was performed using backscattered electron imaging. EDS was used to identify individual phases in the microstructure by their chemical compositions. The ordered nature of the phases could be confirmed only by TEM as the super lattice peaks were too weak to be detected using XRD.

Phase selection during solidification of undercooled $\mathrm{Fe}-\mathrm{Ge}$ melts was also investigated by in situ diffraction with synchrotron radiation at the beamline ID15A of European Synchrotron Radiation Facility (ESRF), Grenoble, France. The experimental setup used is similar to 
that described above and is described elsewhere [13]. At a given angle with respect to the incident beam, energy dispersive, time resolved spectra were collected at time intervals of $3.5 \mathrm{~s}$ during several thermal cycles to capture the temporal evolution of phases during the undercooling process.

\section{Results and discussion}

\subsection{Characterization}

XRD patterns taken from samples solidified at different undercoolings $(\Delta T)$ are plotted in Fig. 3. Samples corresponding to low undercoolings exhibit a phase mixture of all three phases, namely, $\alpha_{2}, \varepsilon$ and $\beta$. Samples solidified at undercoolings above $110 \mathrm{~K}$ contain predominantly $\varepsilon$ phase. The content of other phases is negligible within the detectable limit using XRD. The strong texture noticeable in the patterns is expected since triggered solidification of undercooled melts is directional.

The cooling rate essentially controls the time spent by the sample between the primary recalescence and the solidus/eutectic temperature (plateau time) during which a liquid phase exists. Karma [14] studied the effect of plateau time on the dendrite fragmentation and grain refinement. These studies are based on the observation that the average grain size is comparable to the dendrite trunk radius. Quantitative estimates of dendrite fragmentation require corroboration with the experimentally observed grain size. Since the primary phase undergoes a peritectic transformation in the present system, such a study does not come under the scope of present work. Preliminary experiments performed by dropping the sample onto a copper block indicated that the microstructure is not significantly different from that of the samples cooled off using gas. Fig. 4 shows microstructures of samples solidified at different undercoolings. In the samples solidified at low undercoolings, the phase mixture consists of residual $\alpha_{2}$ phase at the center of the dendrite, $\varepsilon$ phase surrounding the $\alpha_{2}$ phase and inter-dendritic $\varepsilon-\beta$ eutectic. The phases have been identified by their compositions as indicated in Fig. 4(a) and confirmed by TEM as detailed below in this section. The morphology of the phases is typical of a peritectic reaction [7]. The reaction $\mathrm{L}+\alpha_{2} \rightarrow \varepsilon$ indicates that the $\varepsilon$ phase must nucleate at the $\alpha_{2}$ (dendrite) $-\mathrm{L}$ interface and propagate to consume the $\alpha_{2}$ phase. The interface of residual $\alpha_{2}-\varepsilon$ interface is also concave inwards to the centre of the dendrite. The presence of a eutectic reaction $L \rightarrow \varepsilon+\beta$ in the phase diagram close to the present alloy composition indicates the possibility that inter-dendritic liquid solidifies into $\varepsilon-\beta$ eutectic. The morphological appearance of the phases in the microstructure confirms such a typical solidification path.

In the samples solidified at higher undercooling, the microstructure consists predominantly of $\varepsilon$-phase. The peritectic phase morphology described above could not be observed in any of the samples of high undercoolings $(\gg 110 \mathrm{~K})$. Minute traces of $\beta$ phase outlining the original dendritic microstructure and occasionally traces of $\alpha_{2}$ at the centre of the $\varepsilon$ phase could, however, be detected. Samples solidified at undercoolings near and above $160 \mathrm{~K}$ contained only $\varepsilon$-phase. The microstructure also reveals a faceted appearance upon deep etching (not shown here).

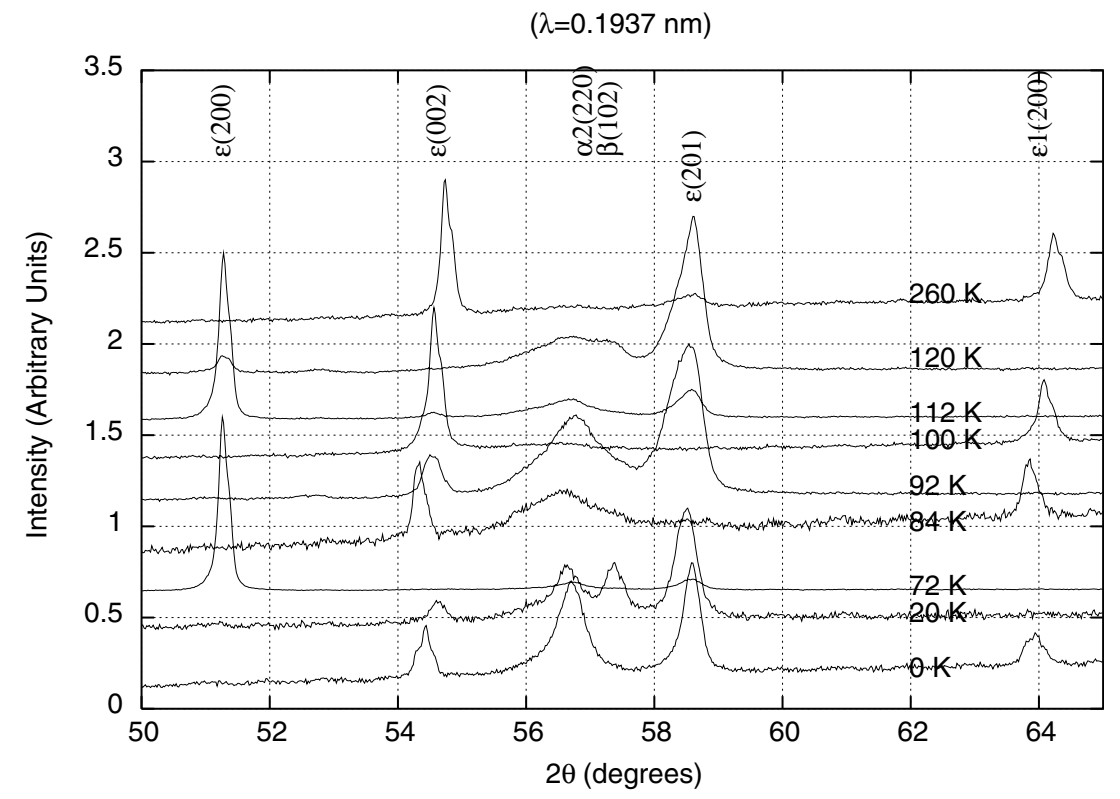

Fig. 3. XRD patterns of samples solidified at different undercoolings. 

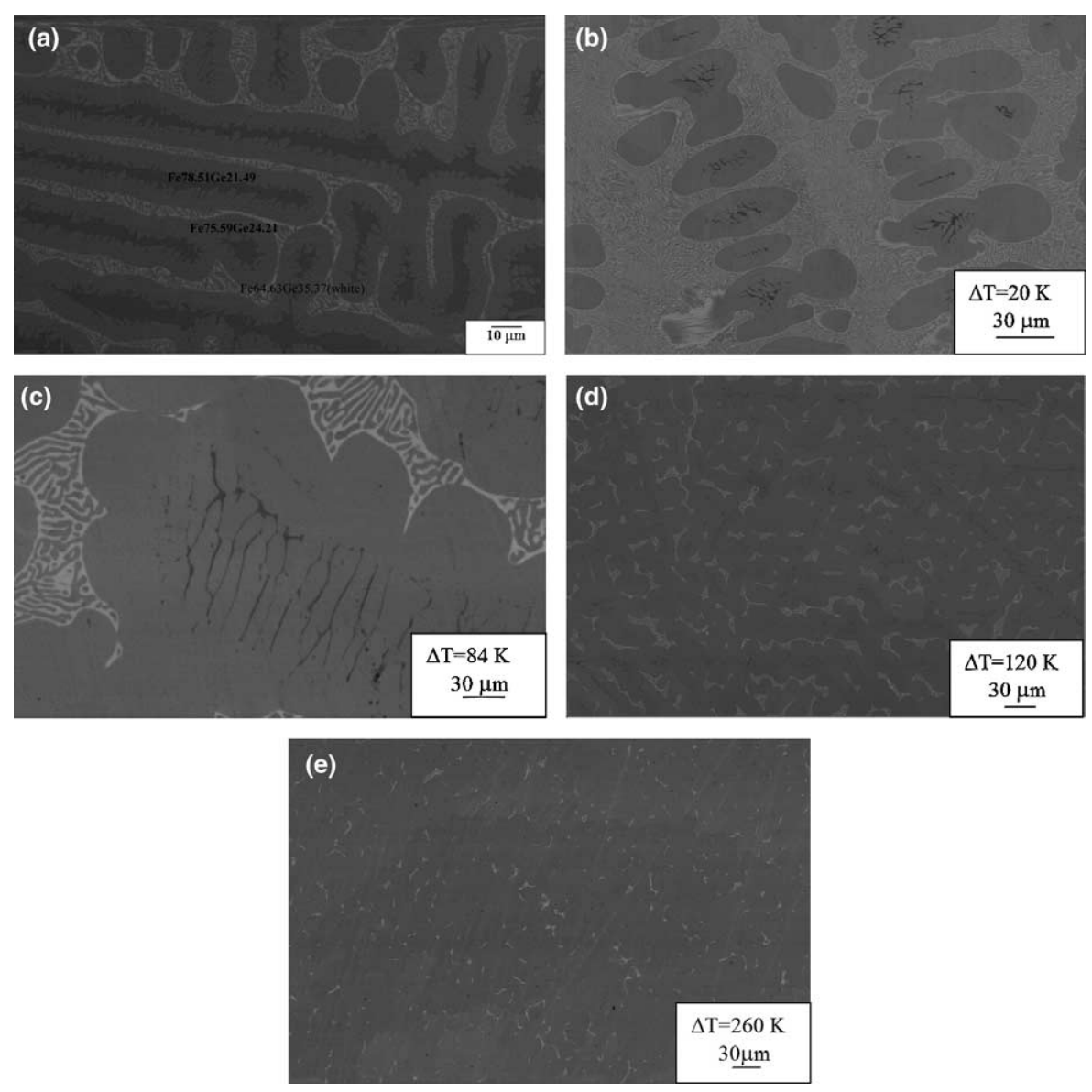

Fig. 4. Microstructures of samples solidified at different undercoolings: (a) as-cast microstructure, illustrating phase identification dark phase is $\alpha$, gray phase is $\varepsilon$ and white phase is $\beta$; (b) $20 \mathrm{~K}$; (c) $84 \mathrm{~K}$; (d) $120 \mathrm{~K}$; (e) $260 \mathrm{~K}$.

\subsubsection{Transmission electron microscopy}

TEM was performed on both non-levitated (as-cast) as well as levitated samples solidified after low as well as high undercoolings. Fig. 5(a) shows the bright field images taken at low magnification. The selected area diffraction taken with the $[1 \overline{1} 00]$ zone axis shows that the final phase is ordered $\mathrm{DO}_{19}$ phase which is metastable at room temperature. A dark field image (Fig. 5(c)) taken using a fundamental reflection $[22 \overline{4} 0]$ shows a network of super-dislocations and no domain structure. However, the dark field image (Fig. 5(d)) taken with a super-lattice reflection $\left[\begin{array}{lll}1 & 1 & 2\end{array}\right]$ ] shows fine anti-phase domains. The analysis performed on the as-cast alloys (not shown here) show that there is no anti-phase domain formation in the $\varepsilon$ phase.

Fig. 6(a) shows the trace amounts of $\alpha_{2}$ that could be detected at the centre of $\varepsilon$ phase. The corresponding selected area diffraction patterns for $\alpha_{2}$ phase (top) and $\varepsilon$ phase (bottom) are shown in Fig. 6(b). Dark field image of $\alpha_{2}$ phase taken using (100) reflection shown in Fig. 6(c) shows that there are no anti-phase domains within $\alpha_{2}$ phase. This indicates that $\alpha_{2}$ solidified primarily as an ordered (B2) phase. The inter-dendritic eutectic has also been confirmed to be between $\beta$ and $\varepsilon$. Fig. 6(d) shows bright field image of eutectic of sample undercooled to $116 \mathrm{~K}$. The corresponding SAD patterns of $\varepsilon$ and $\beta$ phase are shown in inset 1 and inset 2, respectively. Fig. 6(e) shows the dark field image taken [2020] $\varepsilon$ reflection, lighting up $\varepsilon$ lamella. One can also observe the faceted $\beta$ phase.

\subsubsection{In situ diffraction}

Time resolved diffraction was performed to obtain the synchrotron spectra as a function of temperature during heating and cooling cycles of the sample. A total of 20 thermal cycles with undercoolings varying from 13 to $193 \mathrm{~K}$ indicate a constant and reproducible sequence of phase formation. A typical thermal cycle and the synchrotron spectra taken during the solidification are shown in Fig. 7. The first recalescence (sudden increase in temperature) corresponds to the appearance of $\alpha_{2}$ phase and the bulk undercooling experienced by the liquid is $193 \mathrm{~K}$. The spectra taken following this cycle show that the appearance of $\varepsilon$ phase coincides with a change of slope (termed here, the second recalescence). The second recalescence (indicated in Fig. 7 by an arrow) is weak and can be easily mistaken for part of the minor jumps in the pyrometer signal caused due to 

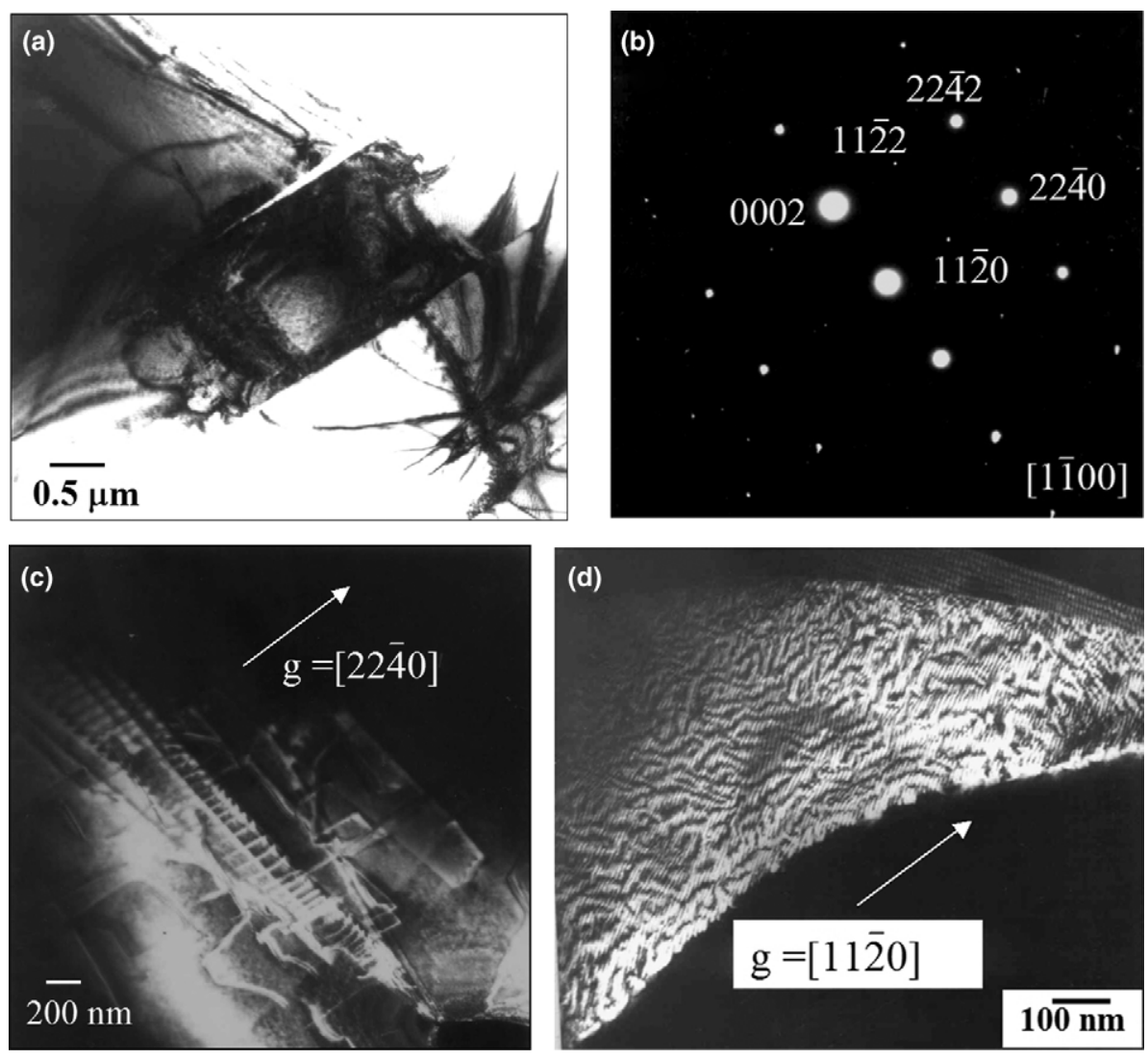

Fig. 5. Transmission electron microscopy of $\mathrm{Fe}+25$ at. $\%$ Ge sample undercooled to $165 \mathrm{~K}$ and solidified: (a) low magnification, bright field image; (b) SAD pattern taken with the [1 100$]$ zone showing ordered nature; (c) dark field image from [22 $\overline{4} 0]$ reflection showing network of superdislocations; (d) dark field image from super-lattice reflection [1 120$]$ showing fine anti-phase domains.

sample motion and oscillations. Time resolved spectra make the identification of the first appearance of $\varepsilon$ phase possible. At temperatures far below the second recalescence, the spectra shows peaks of only the $\varepsilon$ phase. This study confirms that the primary phase to form is $\alpha_{2}$ even at undercoolings larger than $110 \mathrm{~K}$. The time gap between the two recalescence events is about $6 \mathrm{~s}$ here. This time gap between the two recalescence events depends on the flow rate of the protective gas used for cooling, the RF power as well as the undercooling and is found to vary typically between 2 and $12 \mathrm{~s}$.

\subsection{Growth rate measurement}

The growth rate of the primary phase $\alpha_{2}$ measured using the CPS technique is plotted as a function of undercooling $(\Delta T)$ in Fig. 8. The growth rate is sluggish $\left(\sim 0.25 \mathrm{~m} \mathrm{~s}^{-1}\right)$ up to about $\Delta T=110 \mathrm{~K}$ and then increases steadily to nearly $1.5 \mathrm{~m} \mathrm{~s}^{-1}$ at an undercooling of $200 \mathrm{~K}$. The solidification time (time taken by the solidification front to sweep from the nucleation point at the bottom to the top of the sample) is $4-25 \mathrm{~m} \mathrm{~s}$. This value is nearly two orders of magnitude smaller than the time delay between the two recalescence events corresponding to appearance of $\alpha_{2}$ and $\varepsilon$. Hence, the measurement of growth rate corresponds completely to the primary phase $\alpha_{2}$. The error in the measurement of growth rate is estimated to be a maximum of $10 \%$ at all the undercoolings and is indicated by the error bars in the Fig. 8 .

The following analysis presents an attempt to explain the experimentally measured growth rate within the scope of existing theories. The bulk composition of the as-solidified (processed) samples estimated from EDS is between 22 and 25 at.\% while that estimated using electron probe micro analysis (EPMA) is 23.9 at.\%. The loss of Ge as compared to the nominal composition of 25 at. \% chosen for sample preparation is attributed to the arc melting process followed by preferential evaporation loss of Ge during levitation. For the growth rate analysis, we have chosen to compute for both the compositions of 22 at. $\% \mathrm{Ge}$ as well as 25 at. $\%$ Ge to determine the error.

\subsection{Growth rate analysis}

Dendrite growth rate analysis has been carried out using the BCT theory [15]. The bulk undercooling expressed in terms of fractional undercoolings is taken as

$\Delta T=\Delta T_{\mathrm{T}}+\Delta T_{\mathrm{S}}+\Delta T_{\mathrm{R}}+\Delta T_{\mathrm{K}}$. 

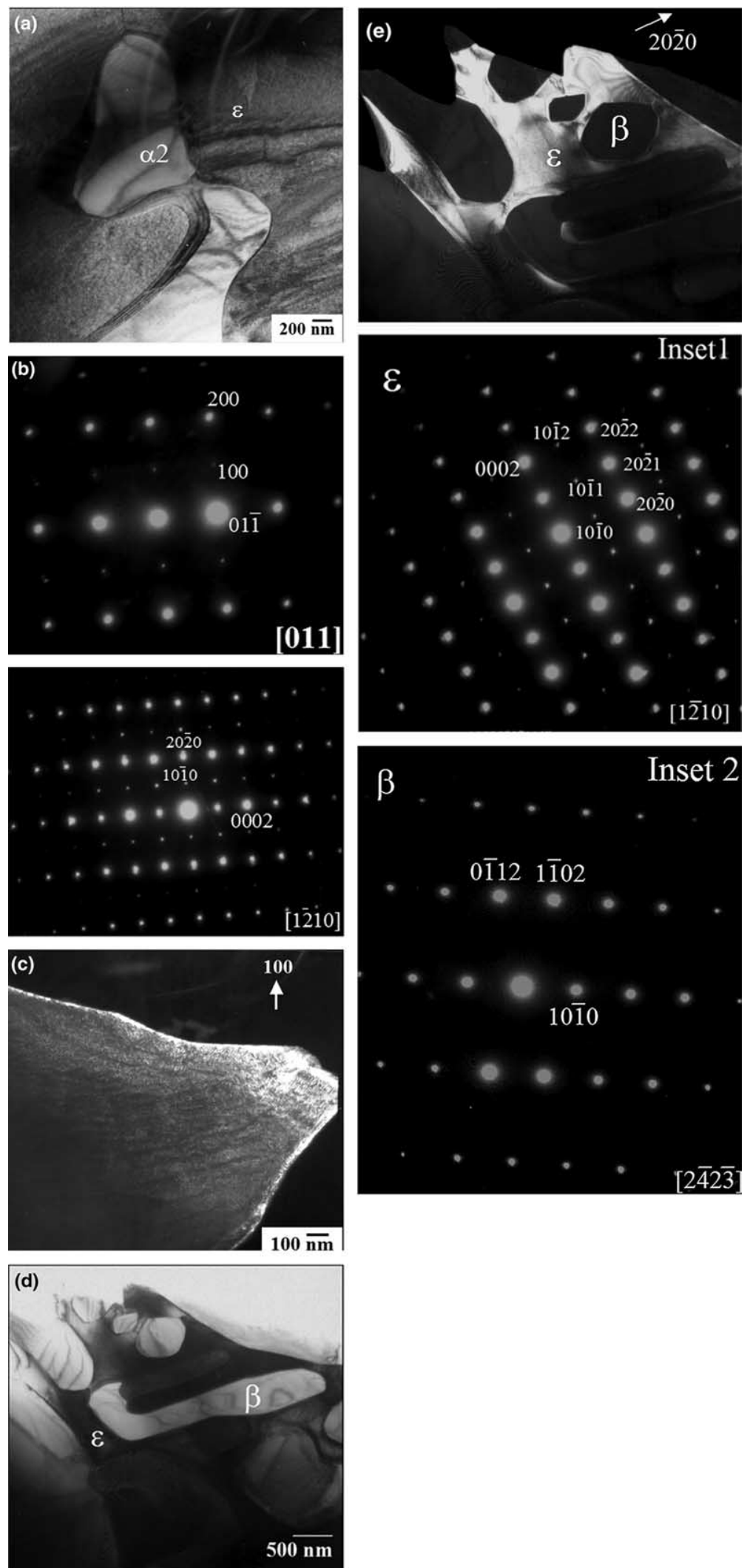

Fig. 6. TEM of sample undercooled to $116 \mathrm{~K}$ : (a) residual trace amounts of $\alpha_{2}$ phase found in the middle of $\varepsilon$ phase; (b) top, SAD pattern from $\alpha_{2}$ showing B2 reflections in [0 1 1] zone, and bottom, SAD pattern from e phase showing DO19 reflections in [1 1210$]$ zone; (c) dark field image of $\alpha_{2}$ phase taken using (100) super lattice reflection showing no anti-phase domains; (d) bright field image of inter-dendritic $\beta-\varepsilon$ eutectic taken with the [1 $1 \overline{2} 10]$ zone axis of $\varepsilon$ phase. The inset 1 shows $[1 \overline{2} 10]$ zone axis pattern of $\varepsilon$ and inset 2 shows $[2 \overline{4} \overline{3}]$ zone axis pattern of $\beta$ phase. 

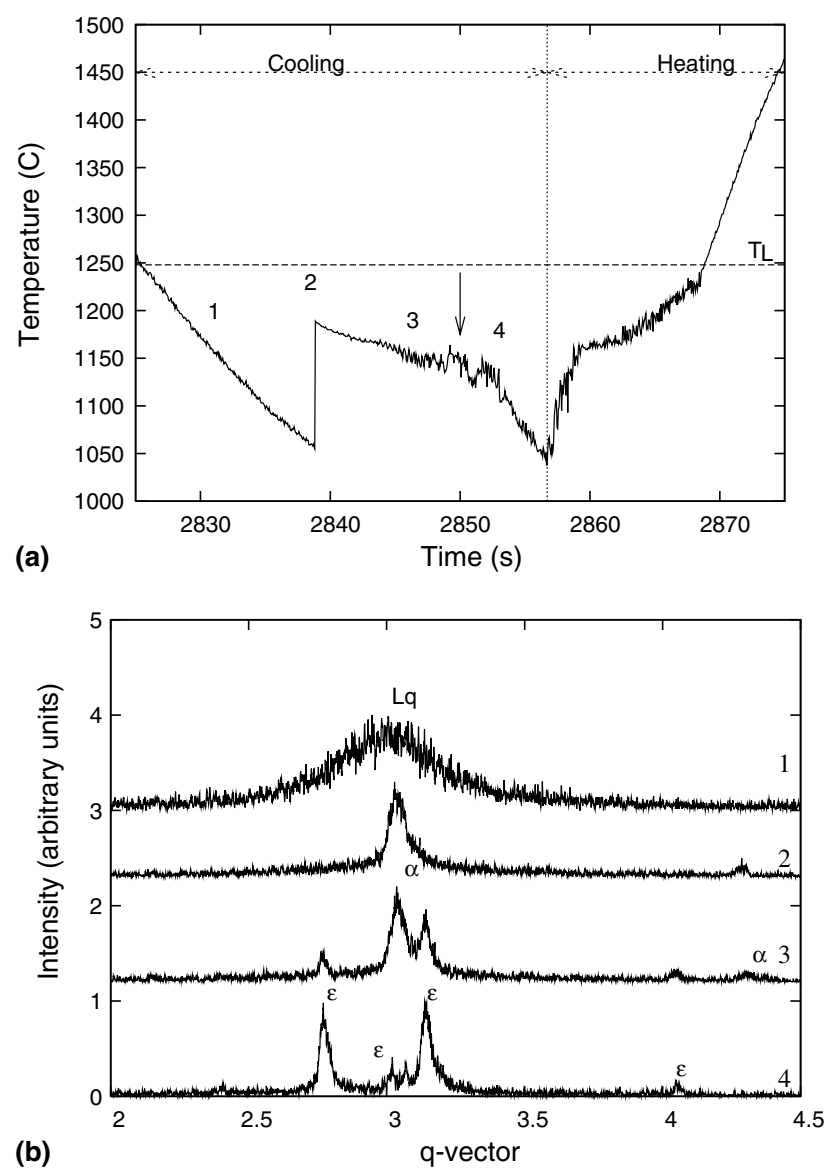

(b)

Fig. 7. (a) Temperature vs. time curve for sample undercooled to 193 $\mathrm{K}$ (top). (b) Synchrotron energy dispersive spectra taken at $2 \theta=2.5^{\circ}$ (bottom) with X-ray intensity as a function of $q$-vector $(2 \pi / d$ where, $d$ is interplanar spacing). The spectra numbered from 1 to 4 are taken at times 2830, 2838, 2846 and $2852 \mathrm{~s}$, respectively, as also indicated in figure above.
The thermal undercooling is given by $\Delta T_{\mathrm{T}}=T_{\mathrm{Q}} I v\left(P_{\mathrm{t}}\right)$ where $T_{\mathrm{Q}}$ is the hypercooling, $I v\left(P_{\mathrm{t}}\right)$ is the Ivantsov function and $P_{\mathrm{t}}=r v / 2 \alpha$ is thermal Peclet number. The solutal undercooling is taken as

$\Delta T_{\mathrm{R}}=m_{0} x_{\mathrm{L}}\left(1-\frac{1+\left(k_{\mathrm{e}}-k\left(1-\log \left(k / k_{\mathrm{e}}\right)\right) / 1-k_{\mathrm{e}}\right.}{1-(1-k) I v\left(P_{\mathrm{c}}\right)}\right)$,

where $m_{0}$ is the liquidus slope, $x_{\mathrm{L}}$ is the composition of liquid, $k_{\mathrm{e}}$ is the equilibrium partition coefficient, $P_{\mathrm{c}}=r v /$ $2 D$ is solutal Peclet number. The effective partition coefficient is $k=\left(k_{\mathrm{e}}+v / v_{\mathrm{D}}\right) /\left(1+v / v_{\mathrm{D}}\right), v$ is the velocity of the interface and $v_{\mathrm{D}}$ is the interface diffusive velocity [16]. $\Delta T_{\mathrm{R}}$ is the curvature undercooling given by $2 T_{\mathrm{M}} \Gamma / r$, $T_{\mathrm{M}}$ is liquidus temperature, $\Gamma$ is the Gibbs-Thomson coefficient, $r$ is the dendrite tip radius given by marginal stability criterion as

$r=\frac{T_{\mathrm{M}} \Gamma / \sigma^{*}}{T_{\mathrm{Q}} P_{\mathrm{t}} \xi_{\mathrm{t}}+\frac{2 m_{0} x_{\mathrm{L}}(k-1)}{1-(1-k) I v\left(P_{\mathrm{c}}\right)}}$.

The stability functions are taken as

$\xi_{\mathrm{t}}=1-\frac{1}{\sqrt{1+\frac{1}{\sigma^{*} P_{\mathrm{t}}^{2}}}} \quad$ and $\quad \xi_{\mathrm{c}}=1+\frac{2 k}{1-2 k-\sqrt{1+\frac{1}{\sigma^{*} P_{\mathrm{c}}^{2}}}}$,

where $\sigma^{*}=1 / 4 \pi^{2}$. The kinetic undercooling $\Delta T_{\mathrm{K}}=v / \mu$ where the kinetic coefficient $\mu$ is given by

$\mu=L v_{\mathrm{s}} / R T_{\mathrm{M}}^{2}$

corresponding to collision limited growth. $L$ is the heat of fusion, $v_{\mathrm{s}}$ is velocity of sound, $R$ is universal gas constant. Eqs. (1) and (3) are solved simulta-

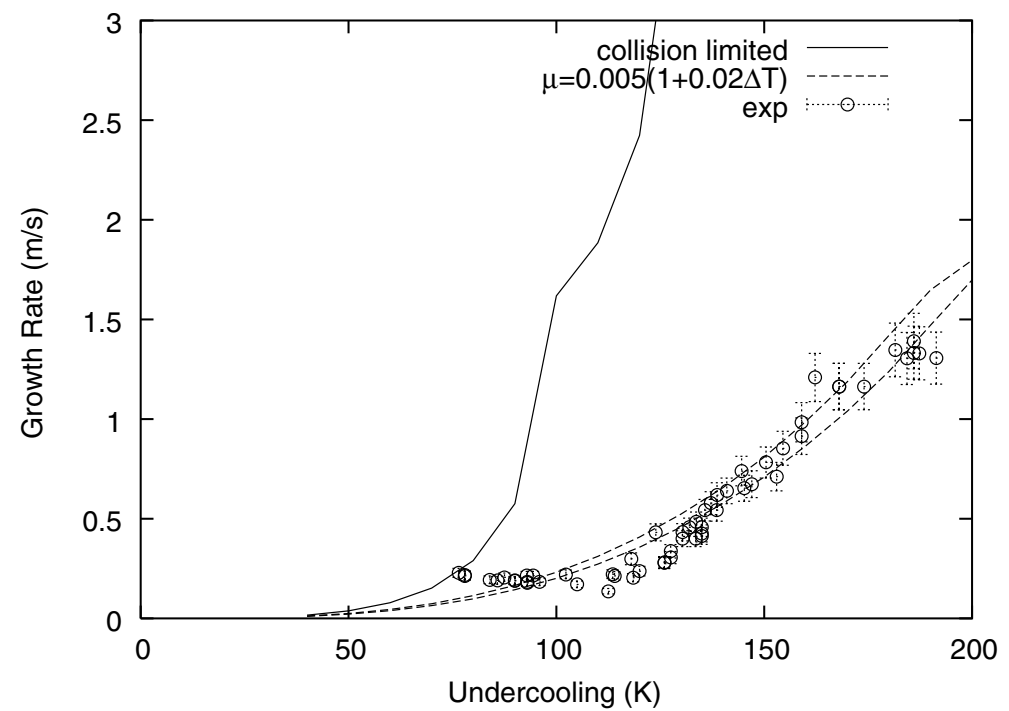

Fig. 8. Experimentally measured growth rate of primary phase $\alpha$. Also super-imposed are the computed growth rates for the assumptions of collision-controlled and diffusion-controlled growth. The upper dashed curve is for $\mathrm{Fe}+22$ at. $\% \mathrm{Ge}$ and the lower dashed curve is for Fe +25 at. $\%$ Ge. 
neously to obtain the growth rate as a function of undercooling. The parameters used are given in Table 1. Where the data is not available, we have estimated the values from corresponding parameters of the pure components [17]. Such an approximation seems reasonable as the primary phase $\alpha_{2}$ for which the growth rate is analyzed, is a solid solution of Fe. The results are plotted in Fig. 8.

As can be noted, the assumption of collision limited growth (Eq. (5)) leads to growth rates significantly higher than the experimentally observed ones. Sluggish growth of ordered intermetallic phases has been observed in several other systems and has been attributed to slower interface attachment kinetics [18,19]. Aziz and Boettinger [18] proposed an expression for interface sluggishness as a function of several thermodynamic parameters apart from $v_{\mathrm{s}}$ and $v_{\mathrm{D}}$. Several studies [2022] have shown that a reduced value of the kinetic coefficient $\mu$ (obtained by replacing $v_{\mathrm{s}}$ with $v_{\mathrm{D}}$ in Eq. (5)) is able to fit the computed growth rate to the experimental data closely. The two dashed curves shown for Fe-22 at.\% $\mathrm{Ge}$ and $\mathrm{Fe}-25$ at.\% Ge lie closely and envelope the experimental data within the estimated error limits. In the present case, no single value of $\mu$ is able to describe the complete experimental data set satisfactorily. Due to the lack of thermodynamic data for this system, we have assumed the kinetic coefficient to be a simple linear function of temperature for this purpose. We were able to fit the computed growth rate to the experimental data when we choose $\mu$ to be small and a function of temperature given by $\mu=0.005[1+0.02 \Delta T]$. The function used corresponds to the effective value of $v_{\mathrm{D}}$ in Eq. (5) ranging from 5 to $15 \mathrm{~m} \mathrm{~s}^{-1}$.

Fig. 9 shows the dendrite tip radius, effective partition coefficient, solid and liquid compositions at the tip and partial undercoolings computed as a function of bulk undercooling for the nominal composition of the alloy. As shown in Fig. 9(b) and (c), the increase in the effec-

Table 1

Values of parameters used in the calculations

\begin{tabular}{lll}
\hline Symbol & Parameter & Value \\
\hline$T_{\mathrm{Q}}$ & Hypercooling $(\mathrm{K})$ & 484.46 \\
$\alpha$ & Thermal diffusivity $\left(\mathrm{m}^{2} \mathrm{~s}^{-1}\right)$ & $1.7 \times 10^{-5}$ \\
$D$ & Solute diffusivity $\left(\mathrm{m}^{2} \mathrm{~s}^{-1}\right)$ & $5.0 \times 10^{-9}$ \\
$\Gamma$ & Gibbs-Thomson coefficient $(\mathrm{K} \mathrm{m})$ & $2.825 \times 10^{-7}$ \\
$v_{\mathrm{s}}$ & Velocity of sound $\left(\mathrm{m} \mathrm{s}^{-1}\right)$ & 2000 \\
$v_{\mathrm{D}}$ & Interface diffusive velocity $\left(\mathrm{m} \mathrm{s}^{-1}\right)$ & 4 \\
$m_{0}$ & Liquidus slope $(\mathrm{K} /$ at. $\%)$ & -17.78 \\
$x_{\mathrm{L}}$ & Liquid composition $($ at. $\%)$ & $22-25$ \\
$k_{\mathrm{e}}$ & Equilibrium partition coefficient & $0.7585-0.7726$ \\
$\mu$ & Kinetic coefficient $\left(R T_{\mathrm{M}}^{2} / L\right)(\mathrm{K})$ & $1011-1090$ \\
$\sigma$ & Solid-liquid interfacial energy $\left(\mathrm{J} \mathrm{m}{ }^{-2}\right)$ & 0.337 \\
$m$ & Volumetric heat of fusion $\left(\mathrm{J} \mathrm{m}{ }^{-3}\right)$ & $1.7 \times 10^{9}$ \\
$X_{\text {eu }}$ & Eutectic composition $($ at. $\%)$ & 30 \\
$n$ & Ratio of radii of curvatures of & $\approx 2$ \\
& secondary arm tip and root & \\
\hline
\end{tabular}

tive partition coefficient by the solute trapping process leads to super-saturation of primary $\alpha_{2}$ phase with the composition the same as that of the final $\varepsilon$ phase.

As can be noted from Fig. 9(d), at smaller undercoolings the dendrite growth mechanism is controlled predominantly by solute diffusion. The solutal undercooling is more than $75 \%$ of the bulk undercooling. However, at $\Delta T>110 \mathrm{~K}$, the kinetic contribution to the bulk undercooling becomes comparable to the solutal contribution $(50 \%)$. Thus, the sharp rise in growth rate at $\Delta T>110 \mathrm{~K}$ corresponds to a change in the dendrite growth mechanism from purely solutal to solutal + kinetic controlled. At large undercoolings, the kinetic undercooling is nearly half of the bulk undercooling, implying that interface attachment kinetics play an important role in determining the dendrite growth rate. Such a large contribution of kinetic undercooling or a small kinetic coefficient is a signature of diffusion controlled growth which is sluggish due to chemical ordering required during the interface attachment process. This is in agreement with the residual primary phase analyzed using TEM being ordered $\alpha_{2}(\mathrm{~B} 2)$ phase.

\subsection{Phase pure microstructure at high undercoolings}

The microstructure of samples solidified at undercoolings larger than $110 \mathrm{~K}$ show nearly phase-pure $\varepsilon$. The negligible amount of residual $\alpha_{2}$ phase and progressive disappearance of inter-dendritic eutectic phase in these samples indicate that for samples solidified at undercoolings larger than $\Delta T>110 \mathrm{~K}$ there are three possible solidification paths. The first possibility is that the $\varepsilon$ phase nucleated directly from the undercooled melt and grew to form the final microstructure. This is ruled out as the in situ diffraction shows clearly that at all the undercoolings (up to $190 \mathrm{~K}$ ) the primary phase to nucleate is $\alpha_{2}$.

The second possibility is that the peritectic reaction proceeded to completion. Since the peritectic reaction involves diffusion of solute through the solid phase, one may estimate the width of the primary solid phase after coarsening and compare it with the diffusion length to consider this possibility. The following simplified expression taken from Kirkwood [23] is shown (e.g., by Chen and Kattamis [24]) to provide a reasonable estimate of secondary arm spacing $\left(\lambda_{\mathrm{f}}\right)$ during solidification.

$\lambda_{\mathrm{f}}^{3}=-\frac{64 \sigma T D}{H m_{0}\left(1-k_{\mathrm{e}}\right)}\left(2-\frac{1}{n}\right) \frac{\ln \left(X_{\mathrm{eu}} / X_{\mathrm{L}}\right)}{X_{\mathrm{eu}}-X_{\mathrm{L}}} t_{\mathrm{f}}$.

The value of the secondary spacing according to the expression depends crucially on the time of solidification $t_{\mathrm{f}}$. The secondary arms evolve for a long time after the primary stem has grown. Thus, the time $t_{\mathrm{f}}$ for estimating the secondary spacing should realistically be close to the 

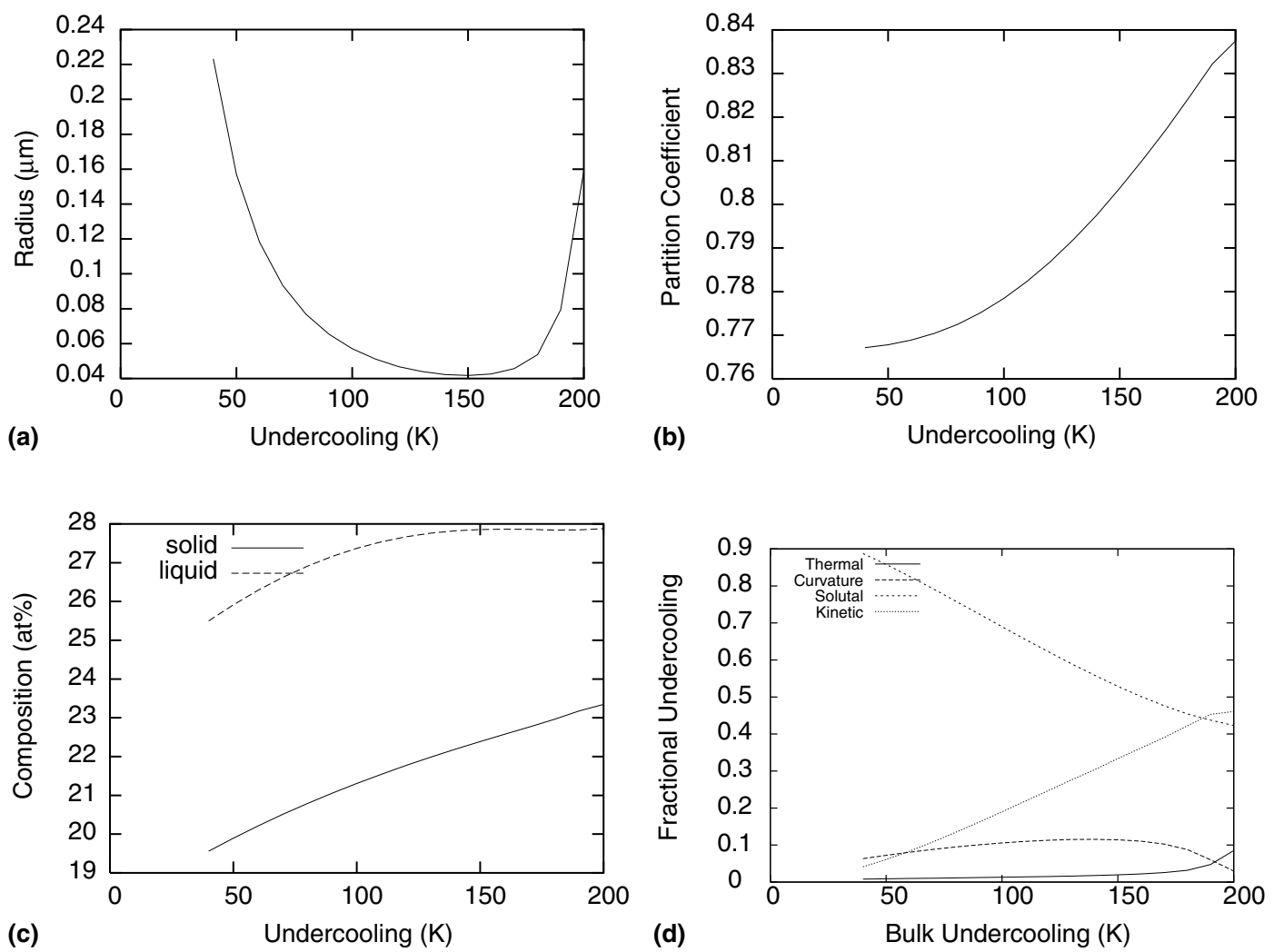

Fig. 9. (a) Dendrite tip radius; (b) effective partition coefficient; (c) solid and liquid composition at the dendrite tip and (d) partial undercoolings as a function of bulk undercooling.

plateau duration ( $t_{\mathrm{p}}$ as indicated in Fig. 2). This results in the lower limit of time available for coarsening to be $10 \mathrm{~s}$. Using this as $t_{\mathrm{f}}$ the estimation yields a secondary arm spacing of $23 \mu \mathrm{m}$. This value is close to the observed values from the microstructures. Taking the diffusivity of $\mathrm{Ge}$ in body-centered cubic (bcc) $\mathrm{Fe}$ at 1435 as $7.033 \times 10^{-13} \mathrm{~m}^{2} \mathrm{~s}^{-1}$ [17], and the maximum time duration between first appearance of $\varepsilon$ phase till complete disappearance of $\alpha$ phase to be about $10 \mathrm{~s}$, leads to a diffusion distance of $2.6 \mu \mathrm{m}$. Thus, the diffusion distance in solute is much smaller than secondary arm spacing suggesting that the peritectic reaction limited by solute diffusion in solid phase is not likely to go to completion.

A third possibility is that the peritectic reaction is suppressed and the formation of $\varepsilon$ phase is by a fast solid-state transformation from $\alpha_{2}$. Though direct evidence is difficult to obtain in the present case, the following arguments point towards this possibility. Growth rate analysis shows that at high undercoolings the primary phase $\alpha_{2}$ solidifies at a composition nearly the same as that of $\varepsilon$ due to solute trapping (Fig. 9(c)) thus requires no diffusion for a solid-state transformation. The disappearance of inter-dendritic eutectic is also a consequence of reduced partitioning. Transformations such as bcc $\rightarrow$ hcp (hcp, hexagonal close-packed) are frequently observed and are usually massive/martensitic in nature [25]. The fine anti-phase domains in $\varepsilon$ phase
(Fig. 5(d)) indicate that such a solid state transformation from $\alpha_{2}$ could be to an initially disordered form (hcp) of $\varepsilon$ phase that later orders to $\mathrm{DO}_{19}$. Within the scope of present analysis, we suggest that at high undercoolings peritectic reaction is suppressed and $\varepsilon$ phase forms via a solid state transformation.

\section{Conclusions}

Solidification of peritectic $\mathrm{Fe}-\mathrm{Ge}$ alloys as a function of undercooling is studied using electromagnetic levitation technique with the following conclusions.

1. In situ diffraction indicates that direct nucleation of the peritectic phase from the undercooled liquid has not taken place. The primary phase to nucleate is always the ordered phase $\alpha_{2}$.

2. Growth rate of the primary phase is sluggish up to $110 \mathrm{~K}$ and then increases sharply. The growth kinetics are explained within the scope of BCT theory using a low kinetic coefficient that increases linearly with undercooling.

3. The predominant phase in the final microstructure is the $\varepsilon$ phase which is metastable at room temperature. Samples solidified at high undercoolings $(\gg 110 \mathrm{~K}$ ) showed nearly phase-pure microstructure. 
4. Peritectic reaction is suppressed and the final phase $\varepsilon$ is suggested to have formed from primary $\alpha_{2}$ phase via a solid state transformation.

\section{Acknowledgements}

Financial support from a joint project between DST, India and BMBF, Germany is gratefully acknowledged. One of the authors (GP) acknowledges AvH Foundation for research fellowship.

\section{References}

[1] Herlach Dieter M. Mater Sci Eng Reports R 1994;12:177-272.

[2] Enoki H, Ishida K, Nishizawa T. Metall Trans 1987;18A:949.

[3] Predel B, Frebel M. Z Metall 1972;63:393.

[4] Raviprasad K, Ranganathan S, Chattopadhyay K. Scripta Metall Mater 1992;26:467.

[5] Biswas K, Phanikumar G, Chattopadhyay K, Volkmann T, Funke O, Holland-Moritz D. Mater Sci Eng A 2004;375$377 \mathrm{C}: 80-3$.

[6] Ngan AHW, Jones IP, Smallman RE. Mater Sci Eng A 1992;153:387.

[7] Mryasov ON, Gornostyrev YuN, van Schlifgaarde M, Freeman AJ. Acta Mater 2002;50(2002):4545.
[8] Kerr HW, Kurz W. Int Mater Rev 1996;41:129.

[9] Chen QZ, Ngan AHW, Duggan BJ. Intermetallics 1998; 6:105.

[10] Schleip E, Willnecker R, Herlach DM, Görler GP. Mater Sci Eng 1988;98:39.

[11] Eckler K, Kratz M, Egry I. Rev Sci Instrum 1993;64(9): 2639.

[12] Eckler K, Herlach DM. Mater Sci Eng A 1994;178:159.

[13] Notthoff C, Franz H, Hanfland M, Herlach DM, Holland-Mority D, Petry W. Rev Sci Instrum 2000;71:3791.

[14] Karma A. Int J Non-Eq Proc 1998;11:201-33.

[15] Boettinger WJ, Corriell SR, Trivedi R. In: Mehrabian R, Parrish PA, editors. Proceedings of the 4th conference on rapid solidification processing: principles and technologies, 1987. Baton Rouge (LA): Claitors; 1987. p. 13.

[16] Aziz MJ, Kaplan T. Acta Metall 1998;30:2335.

[17] Brandes EA. Smithells metals reference book. 6th ed. London: Butterworth; 1983.

[18] Barth M, Wei B, Herlach DM. Phys Rev B 1995;51:3422.

[19] Aziz MJ, Boettinger WJ. Acta Metall Mater 1994;42:527.

[20] Barth M, Wei B, Herlach DM, Feuerbacher B. Mater Sci Eng A 1994;178:305.

[21] Eckler K, Gärtner F, Assadi H, Norman AF, Greer AL, Herlach DM. Mater Sci Eng A 1997;226-228:410.

[22] Goetzinger R, Barth M, Herlach DM, Hunziker O, Kurz W. Mater Sci Eng A 1997;226-228:415.

[23] Kirkwood DH. Mater Sci Eng 1985;73:L1.

[24] Chen M, Kattamis TZ. Mater Sci Eng A 1998;247:239-47.

[25] Nishitani Shigeto R. Hitoshi Kawabe and Masato Aoki. Mater Sci Eng A 2001;312(1-2):77. 